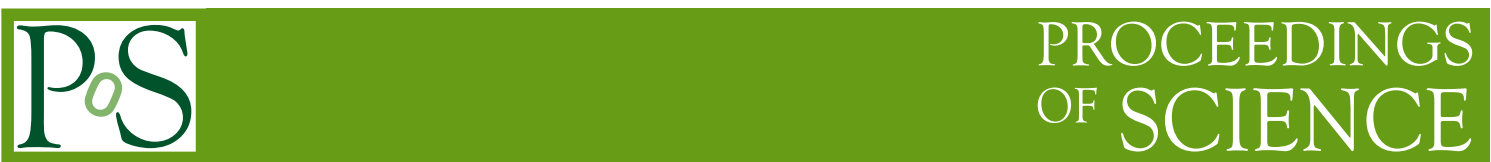

\title{
New physics analysis of some $b$-baryon decays
}

\section{P Haritha ${ }^{a, *}$ and Barilang Mawlong ${ }^{a}$}

${ }^{a}$ School of Physics, University of Hyderabad, Hyderabad - 500046, India.

E-mail: harithacp2010@gmail.com, barilang05@gmail.com

Measurements in the $b \rightarrow c \tau^{-} \bar{v}_{\tau}$ transitions suggest violation of lepton flavor universality (LFU). Assuming the flavor anomalies are due to new physics (NP) beyond the Standard Model (SM), we analyse the semileptonic decays of some heavy $b$-baryons to $c$-baryons, $\Sigma_{b} \rightarrow \Sigma_{c} \tau^{-} \bar{v}_{\tau}$ and $\Sigma_{b} \rightarrow \Sigma_{c}^{*} \tau^{-} \bar{v}_{\tau}$, which are mediated by $b \rightarrow c \tau^{-} \bar{v}_{\tau}$ transitions. Using a general effective Hamiltonian which includes both SM and NP contributions, we study and discuss the effects of the new contributions on the semileptonic $q^{2}$ spectra, such as the differential decay rate, ratio of branching fractions, forward-backward asymmetry of the charged lepton, convexity parameter and longitudinal polarization of the charged lepton in various new physics scenarios.

*** The European Physical Society Conference on High Energy Physics (EPS-HEP2021), ***

*** 26-30 July $2021 * * *$

*** Online conference, jointly organized by Universität Hamburg and the research center DESY ***

${ }^{*}$ Speaker 


\section{Introduction}

Recent experimental studies in the $B$ sector show deviations from the standard model (SM) expectations. The world average values by HFLAV [1] for the ratio of branching fractions $R_{D}=$ $\Gamma\left(B \rightarrow D \tau \bar{v}_{\tau}\right) / \Gamma\left(B \rightarrow D l \bar{v}_{l}\right)=0.340 \pm 0.027 \pm 0.013$ and $R_{D^{*}}=\Gamma\left(B \rightarrow D^{*} \tau \bar{v}_{\tau}\right) / \Gamma(B \rightarrow$ $\left.D^{*} l \bar{v}_{l}\right)=0.295 \pm 0.011 \pm 0.008$, exceed their SM predictions by $1.4 \sigma$ and $2.5 \sigma$, respectively. The LHCb measurement of the ratio $R_{J / \psi}=\Gamma\left(B_{c} \rightarrow J / \psi \tau \bar{v}_{\tau}\right) / \Gamma\left(B_{c} \rightarrow J / \psi \mu \bar{v}_{\mu}\right)=0.71 \pm 0.17 \pm 0.18$ [2] is about $2 \sigma$ larger than its SM prediction. The observed flavor anomalies suggest the presence of new physics (NP) beyond the SM in $b$-hadron semitauonic decays and motivates the study of similar decay modes governed by $b \rightarrow c \tau v_{\tau}$ quark transitions. Measurement of the $\tau$ polarization $P_{\tau}^{D^{*}}=-0.38 \pm 0.51_{-0.16}^{+0.21}$ [3] and the $D^{*-}$ polarization $F_{L}^{D^{*}}=0.60 \pm 0.08 \pm 0.04$ [4] by the Belle collaboration also furnish additional aspects to analyse NP in these decay modes. In this work, we study and analyse the semileptonic decay of $\Sigma_{b} \rightarrow \Sigma_{c}^{(*)} \tau \bar{v}_{\tau}$ in both SM and various NP scenarios following a model-independent approach. The $\Sigma_{b}$ baryon predominantly decays strongly and thus it may be difficult to measure their weak branching fraction. However, it is worth studying these decay modes as they can be more sensitive to virtual new particles and any deviation from SM prediction will be a clear signature of NP [5].

\section{Theoretical Framework}

The most general effective Hamiltonian for decays mediated by $b \rightarrow c l v_{l}$ quark transitions is given by [6]

$$
\mathcal{H}_{e f f}=\frac{4 G_{F}}{\sqrt{2}} V_{c b}\left[\left(1+C_{V_{L}}\right) O_{V_{L}}+C_{V_{R}} O_{V_{R}}+C_{S_{R}} O_{S_{R}}+C_{S_{L}} O_{S_{L}}+C_{T} O_{T}\right]+\text { h.c. },
$$

where $G_{F}$ is the Fermi coupling constant, $V_{c b}$ is the CKM matrix element, and $C_{V_{L, R}}, C_{S_{L, R}}, C_{T}$ denote vector, scalar, tensor NP couplings, respectively. The fermionic operators are defined as $O_{V_{L, R}}=\left(\bar{c} \gamma^{\mu} b_{L, R}\right)\left(\bar{l}_{L} \gamma_{\mu} v_{l_{L}}\right), O_{S_{L, R}}=\left(\bar{c} b_{L, R}\right)\left(\bar{l}_{R} v_{l_{L}}\right)$ and $O_{T}=\left(\bar{c} \sigma^{\mu v} b_{L}\right)\left(\bar{l}_{R} \sigma_{\mu \nu} v_{l_{L}}\right)$. In this work, we consider only vector and scalar type of interactions; we assume the neutrino is left-handed and the NP couplings are real. As the vector operator with a right-handed quark current does not contribute to LFU violation [7, 8], we do not include the effects of $C_{V_{R}}$ in our analysis.

The form factors which parametrize the hadronic matrix elements of vector and axial vector currents for $\Sigma_{b} \rightarrow \Sigma_{c}^{(*)} \tau^{-} \bar{v}_{\tau}$ decays have been taken from [9].

\section{1 $q^{2}$ - dependent observables}

The differential decay rate for $\Sigma_{b} \rightarrow \Sigma_{c}^{(*)} \tau^{-} \bar{v}_{\tau}$ expressed in terms of helicity amplitudes is given by [10],

$$
\frac{d \Gamma}{d q^{2}}=\frac{G_{F}^{2}\left|V_{c b}\right|^{2} q^{2}\left|\mathbf{p}_{B_{c}}\right|}{192 \pi^{3} m_{B_{b}}^{2}}\left(1-\frac{m_{l}^{2}}{q^{2}}\right)^{2} \mathcal{H}_{\frac{1}{2} \rightarrow \frac{1}{2}\left(\frac{3}{2}\right)},
$$

where $\mathcal{H}_{\frac{1}{2} \rightarrow \frac{1}{2}\left(\frac{3}{2}\right)}$ denote the total helicity amplitudes which contain both SM and NP contributions. Their explicit forms can be found in [10].

We also consider other interesting $q^{2}$-dependent observables such as the ratio of branching fractions $R\left(q^{2}\right)$, forward-backward asymmetry of the charged lepton $A_{F B}^{\tau}\left(q^{2}\right)$, convexity parameter $C_{F}^{\tau}\left(q^{2}\right)$ and longitudinal polarization of the charged lepton $P_{L}^{\tau}\left(q^{2}\right)$. 


\section{New Physics sensitivity}

We constrain the new couplings $C_{V_{L}}, C_{S_{L, R}}$ using the observables $R_{D^{(*)}}, R_{J / \psi}, F_{L}^{D^{*}}$ and $P_{\tau}^{D^{*}}$. The allowed NP parameter space is obtained by imposing a $1 \sigma$ constraint coming from the experimental measurements of these observables. We also impose a $30 \%$ constraint coming from the upper bound of $\mathcal{B}\left(B_{c}^{+} \rightarrow \tau^{+} v_{\tau}\right)$ [11]. Considering one coupling at a time, we obtain the best-fit values presented in Table 1 by performing a $\chi^{2}$ fit. The $\chi^{2}$ function is defined as [7]

$$
\chi^{2}\left(C_{k}\right)=\sum_{i j}^{N_{\text {obs }}}\left[O_{i}^{\text {exp }}-O_{i}^{t h}\left(C_{k}\right)\right] C_{i j}^{-1}\left[O_{j}^{\text {exp }}-O_{j}^{t h}\left(C_{k}\right)\right],
$$

where $O_{i}^{\text {exp }}$ denote the measured value of the observables, $O_{i}^{t h}\left(C_{k}\right)$ are the theoretical predictions for the observables with the new couplings $C_{k}$, and $C$ is the covariance matrix which takes into account the correlation of $R_{D}$ and $R_{D^{(*)}}$.

\begin{tabular}{ccc}
\hline NP coupling & Best-fit value & $1 \sigma$ range \\
\hline$C_{V_{L}}$ & 0.069 & {$[0.052,0.087]$} \\
$C_{S_{L}}$ & 0.113 & {$[0.065,0.158]$} \\
$C_{S_{R}}$ & 0.131 & {$[0.089,0.171]$} \\
\hline
\end{tabular}

Table 1: Best-fit values of the NP couplings.

The $q^{2}$-dependence of the relevant observables for $\Sigma_{b} \rightarrow \Sigma_{c}^{(*)} \tau^{-} \bar{v}_{\tau}$ decays are reported in Figs. 1 and 2. We present predictions in both the SM case and in the presence of various new couplings. The $d \Gamma / d q^{2}$ observable is enhanced over the whole $q^{2}$ region in presence of both vector and scalar couplings for $\Sigma_{b} \rightarrow \Sigma_{c} \tau^{-} \bar{v}_{\tau}$. In presence of scalar couplings, $d \Gamma / d q^{2}$ displays only a tiny deviation for the $\Sigma_{b} \rightarrow \Sigma_{c}^{*} \tau^{-} \bar{v}_{\tau}$ mode. The ratio $R_{\Sigma_{c}}\left(q^{2}\right)$ shows a deviation from the SM in higher $q^{2}$ region for $\Sigma_{b} \rightarrow \Sigma_{c} \tau^{-} \bar{v}_{\tau}$ decay and behaves SM-like in case of the $C_{V_{L}}$ coupling, as the Lorentz structure of the operator $O_{V_{L}}$ is the same as the SM. Similar behaviour is observed for the other observables with respect to $C_{V_{L}}$. For $\Sigma_{b} \rightarrow \Sigma_{c}^{*} \tau^{-} \bar{v}_{\tau}$ decay, a tiny deviation is observed for $R_{\Sigma_{c}}\left(q^{2}\right)$ in presence of scalar couplings. The forward-backward asymmetry $A_{F B}^{\tau}\left(q^{2}\right)$ shows almost zero deviation from $\mathrm{SM}$ in the presence of scalar couplings for $\Sigma_{b} \rightarrow \Sigma_{c} \tau^{-} \bar{v}_{\tau}$. For $\Sigma_{b} \rightarrow \Sigma_{c}^{*} \tau^{-} \bar{v}_{\tau}$ mode, $A_{F B}^{\tau}\left(q^{2}\right)$ has a SM zero crossing point at $q^{2} \approx 6.6 \mathrm{GeV}^{2}$. This point shifts to a lower $q^{2}$ value with $C_{S_{L}}$ coupling and it shifts to a higher $q^{2}$ value with $C_{S_{R}}$ coupling. The convexity parameter $C_{F}^{\tau}\left(q^{2}\right)$ displays a distinguishable deviation from the SM prediction in case of scalar couplings for both decay modes. For $\Sigma_{b} \rightarrow \Sigma_{c} \tau^{-} \bar{v}_{\tau}$ decay, the polarization $P_{L}^{\tau}\left(q^{2}\right)$ shows a clear deviation from the SM with $C_{S_{L}}$ and $C_{S_{R}}$ couplings. We observe a SM zero crossing in $P_{L}^{\tau}\left(q^{2}\right)$ at $q^{2} \approx 3.5 \mathrm{GeV}^{2}$ for $\Sigma_{b} \rightarrow \Sigma_{c}^{*} \tau^{-} \bar{v}_{\tau}$ decay and the zero crossing shifts to lower and higher $q^{2}$ values with $C_{S_{L}}$ and $C_{S_{R}}$ couplings, respectively.

\section{Conclusion}

We have analyzed the semileptonic decay modes $\Sigma_{b} \rightarrow \Sigma_{c} \tau^{-} \bar{v}_{\tau}$ and $\Sigma_{b} \rightarrow \Sigma_{c}^{*} \tau^{-} \bar{v}_{\tau}$ within the SM and beyond. We have presented predictions for some $q^{2}$ - dependent observables in various 

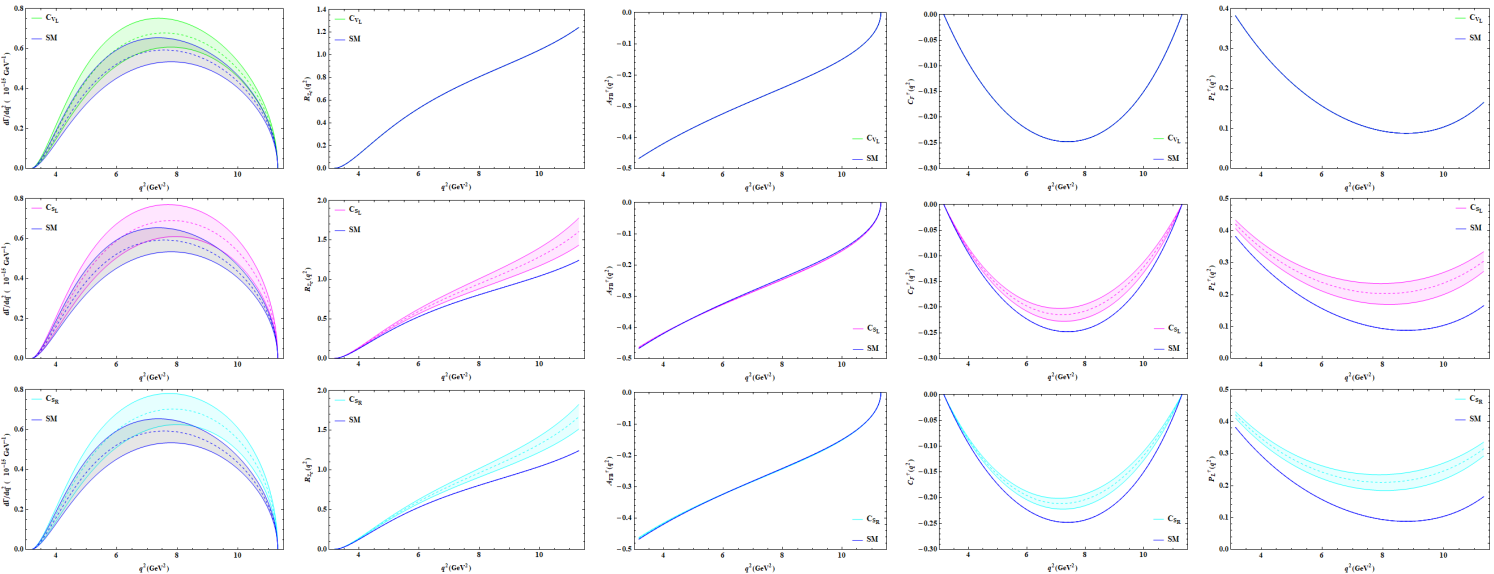

Figure 1: The $q^{2}$-dependency of various observables for $\Sigma_{b} \rightarrow \Sigma_{c} \tau^{-} \bar{v}_{\tau}$ decay mode in the presence of vector and scalar NP couplings.
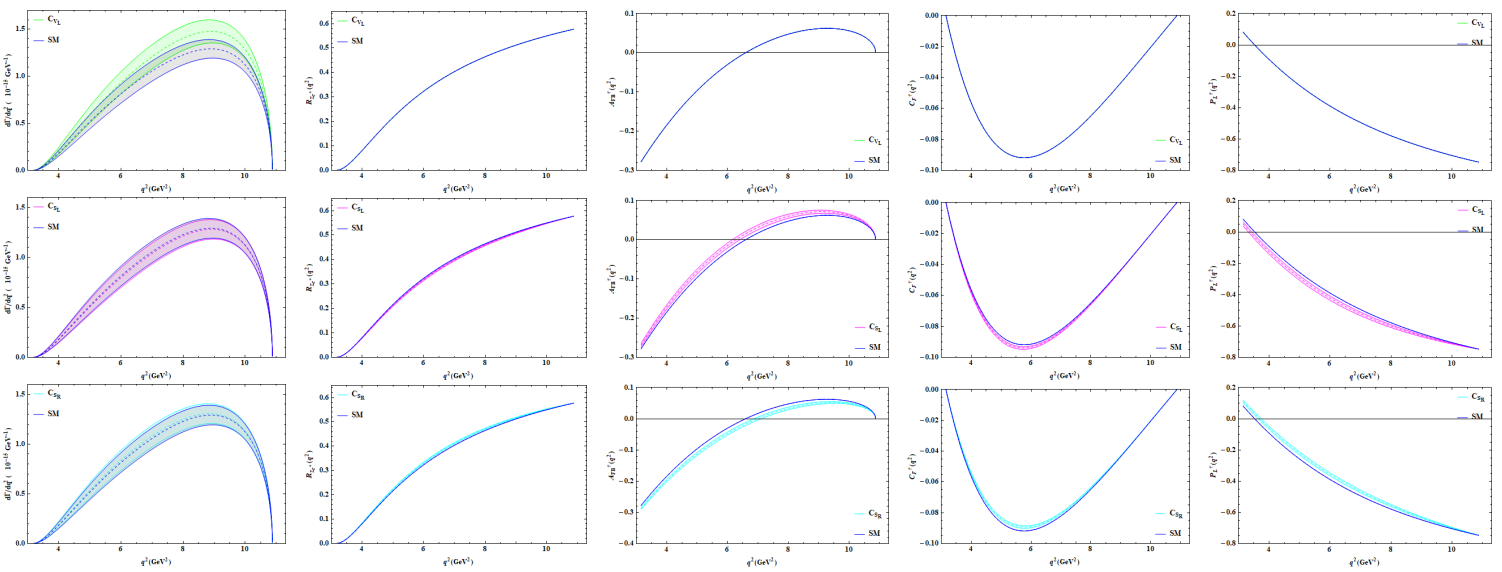

Figure 2: The $q^{2}$-dependency of various observables for $\Sigma_{b} \rightarrow \Sigma_{c}^{*} \tau^{-} \bar{v}_{\tau}$ decay mode in the presence of vector and scalar NP couplings.

NP scenarios. We found NP sensitivity of the observables in most of the scenarios with vector or scalar couplings considered here. While the differential decay rate appears to be more sensitive to NP for the $\Sigma_{b} \rightarrow \Sigma_{c} \tau^{-} \bar{v}_{\tau}$ decay mode, other observables such as the forward-backward asymmetry of the charged lepton shows a distinguishable behaviour in case of the $\Sigma_{b} \rightarrow \Sigma_{c}^{*} \tau^{-} \bar{v}_{\tau}$ mode. The semileptonic decay modes of $b$-baryons, which are of half-integer spin, provide a complementary environment for NP sensitivity search to that of the meson modes. Hence, their study can be helpful in identifying and determining the nature of NP.

\section{References}

[1] HFLAV collaboration, Averages of b-hadron, c-hadron, and $\tau$-lepton properties as of 2018, Eur. Phys. J. C 81 (2021) 226 [1909. 12524].

[2] LНСв collaboration, Measurement of the ratio of branching fractions $\mathcal{B}\left(B_{c}^{+} \rightarrow J / \psi \tau^{+} v_{\tau}\right) / \mathcal{B}\left(B_{c}^{+} \rightarrow J / \psi \mu^{+} v_{\mu}\right)$, Phys. Rev. Lett. 120 (2018) 121801 
[1711.05623].

[3] BeLle collaboration, Measurement of the $\tau$ lepton polarization and $R\left(D^{*}\right)$ in the decay $\bar{B} \rightarrow D^{*} \tau^{-} \bar{v}_{\tau}$, Phys. Rev. Lett. 118 (2017) 211801 [1612.00529].

[4] Belle collaboration, Measurement of the $D^{*-}$ polarization in the decay $B^{0} \rightarrow D^{*-} \tau^{+} v_{\tau}$, in 10th International Workshop on the CKM Unitarity Triangle, 3, 2019 [1903.03102].

[5] H.-W. Ke, N. Hao and X.-Q. Li, Revisiting $\Lambda_{b} \rightarrow \Lambda_{c}$ and $\Sigma_{b} \rightarrow \Sigma_{c}$ weak decays in the light-front quark model, Eur. Phys. J. C 79 (2019) 540 [1904.05705].

[6] C. Murgui, A. Peñuelas, M. Jung and A. Pich, Global fit to $b \rightarrow c \tau v$ transitions, JHEP 09 (2019) 103 [1904.09311].

[7] M. Blanke, A. Crivellin, S. de Boer, T. Kitahara, M. Moscati, U. Nierste et al., Impact of polarization observables and $B_{c} \rightarrow \tau v$ on new physics explanations of the $b \rightarrow c \tau v$ anomaly, Phys. Rev. D 99 (2019) 075006 [1811.09603].

[8] R.-X. Shi, L.-S. Geng, B. Grinstein, S. Jäger and J. Martin Camalich, Revisiting the new-physics interpretation of the $b \rightarrow c \tau v$ data, JHEP 12 (2019) 065 [1905 . 08498].

[9] D. Ebert, R.N. Faustov and V.O. Galkin, Semileptonic decays of heavy baryons in the relativistic quark model, Phys. Rev. D 73 (2006) 094002 [hep-ph/0604017].

[10] T. Gutsche, M.A. Ivanov, J.G. Körner, V.E. Lyubovitskij, P. Santorelli and C.-T. Tran, Analyzing lepton flavor universality in the decays $\Lambda_{b} \rightarrow \Lambda_{c}^{(*)}\left(\frac{1}{2}^{ \pm}, \frac{3}{2}^{-}\right)+\ell \bar{v}_{\ell}$, Phys. Rev. D 98 (2018) 053003 [1807 . 11300].

[11] R. Alonso, B. Grinstein and J. Martin Camalich, Lifetime of $B_{c}^{-}$Constrains Explanations for Anomalies in $B \rightarrow D^{(*)} \tau v$, Phys. Rev. Lett. 118 (2017) 081802 [1611.06676]. 